\title{
UN MANOMETRE ENREGISTREUR DE PRESSIONS SOUS-MARINES POUR LA DÉTERMINATION DES CARACTÉRISTIQUES DE LA HOULE PAR LA MESURE DES VARIATIONS DE PRESSION EN PROFONDEUR
}

\author{
THE DETERMINATION OF WAVE CHARACTERISTICS BY MEASUREMENTS \\ OF DEEP PRESSURE VARIATIONS \\ English synopsis p. 671
}

par J. VALEMBOIS

Docteur ès Sciences

Ingénieur au Laboratoire National d'Hydraulique - Chatou

\section{INTRODUCTION}

L'appareil que je vais décrire dans cet exposé a été étudié et mis au point cu Laboratoire de Chatou pour la mesure des caractéristiques de la houle. Il a été expérimenté en mer à plusieurs reprises et a donné de bons résultats. Actuellement un manomètre est mouillé dans l'estuairc de la Rance pour des essais de longue durée.

Il est constitué par un manomètre électrique que I'on pose sur le fond à l'endroit voulu et une station enregistreuse à terre, ces deux éléments étant reliés par un câble électrique d̀ 4 conducteurs.

Le manomètre peut être conçu de façon à mesurer les houles, les seiches ou les marées. Le dispositif récepteur est tel qu'il peut enregistrer les indications d'un appareil quelconque (manomètre à houle, marégraphe, thermomètre, mesureur de courant, etc...).

En vue d'éliminer le câble, qui est cher et dont la pose est coûteuse, nous étudions actuellement un enregistreur étanche qui sera mouillé sur le fond à peu de distance du manomètre et pourra fonctionner de façon autonome pendant plusieurs mois.

Cette version de l'appareil résoudra à peu de frais la plupart des problèmes qui nécessitent la mesure des caractéristiques de la houle, mais, pour certains cas particuliers, le manomètre relié à la terre par un câble sera pratiquement seul utilisable.

Si par exemple on veut avoir des données sur la houle attaquant un port, pour étudier la résistance de ses digues ou la configuration de ses bassins, ou bien si l'on veut faire une mesure des caractéristiques moyennes de la houle dans une région où l'on envisage d'utiliser son énergie, le manomètre avec enregistreur autonome annexe conviendra très bien. On le prévoira de taçon à faire un enregistrement de courte durée c) intervalles réguliers (par exemple 20 minutes toutes les six heures). De plus un déclencheur manométrique le mettra en marche pendant les tempêtes, lorsque le creux de la houle dépassera une certaine valeur fixée d̀ l'avance.

Mais si la mesure de la houle a pour but de fournir des éléments de base cux prévisions météorologiques (1), on ne peut pas utiliser un appareil que l'on relève tous les deux ou trois mois. Il faut qu'on ait sous les yeux l'enregistrement à l'instant même où il s'effectue. Dans un cas comme celui-là, deux solutions sont pos-

(1) C'était le cos pendant la guerre, où l'Amirauté anglaise foisait des mesures de houle afin d'avoit des étements de base pour la prévision du creux de la hotle sui les plages de déborquement. 
sibles. Un appareil comme le nôtre, relié à terre par un câble ou un appareil avec retransmișsion par radio. Le second est en définitive beaucoup plus onéreux. D'autre part, tandis que le premier peut fonctionner aussi longtemps que l'on voudra sans être relevé ("), le second devra comporter une source d'énergie autonome qui fatalement s'épuisera au bout d'un temps assez court. Ceci n'est d'ailleurs pas rédhibitoire, beaucoup moins que la nécessité d'avoir un émetteur flottant, excessivement coûteux si l'on veut qu'il résiste aux tempêtes.

Les types d'appareils dont nous venons de parler ne sont utilisés pour la mesure de la houle que depuis peu de temps. Encore maintenant, dans la plupart des cas, on doit se reporter aux estimations des marins, qui sont assez sujettes à caution, tout au moins quant aux creux de houle. Elles ont d'ailleurs donné lieu à des controverses mémorables par leur violence (").

Une règle assez bonne dans bien des cas, mais qu'on ne peut évidemment appliquer à coup sûr, est que le creux estimé à l'œil est environ le double du creux réel $\left({ }^{4}\right)$.

Pour suppléer à l'imprécision des «mesures à l'estime », on a imaginé bien des appareils et cles méthodes. Le problème est relativement facile à résoudre si l'on veut connaître les variations du niveau à la côte, par exemple le long d'un mur de quai. Il se complique pour les mesures faites a quelque distance du rivage. On a utilisé divers procédés, par exemple le trace vague de FROUDE ("), un baromètre très sensible placé sur un bateau (i), la stéréophotographie du plan d'eau ( ${ }^{7}$ ), etc..., mais les seuls appareils permettant jusqu'à maintenant d'obte. nir des enregistrements continus de la houle sont d'une part les sondeurs à ultra-sons placés au fond et envoyant verticalement un faisceau qui se réfléchit à la surfoce, d'autre part les mano-

(2) C'est-ádire en protique oussi longtemps qu'il résistera mécaniquement aux agents c'estructeurs de la mer (courants, micro-organismes, etc...)

(3) DUMONT D'URVILLE ayant rapporté, dans son "Histoire du voyage de découvertes autour du monde ef a la recherche de LA PEROUSE ", en 1826, qu'il avait observé des houles atteignant 100 pieds de creux, soit 33 mètres, ARAGO avait répliqué dans les termes suivants : "Eussé-je été en proie à la plus violente des passions, les travaux de $M$. D'URVILLE ne miauraient pas donné l'occosion de l'exercer et cela en vertu du vieil adage : là où il n'y a rien, le roi perd ses droits ».

(4) L'expérience montre que même des observateurs connaissant cette règle ont tendance à surestimer la hauteur de la houle.

(5) BOUASSE : Houles, rides, seiches et marées, 1924, p. 67. - J. ROUCH : Traité d'Océanographie physique, t. 3,1948, p. 14 .

(6) 1. ROUCH : op.cit, P. 15.

(7) 1. ROUCH : op. cit. p. 17 mètres, qui mesurent les variations de pression développées au fond de la mer par le passage des vagues. On n'obtient d'ailleurs par ces deux procédés que le creux et la période. Si l'on veut connaitre la direction de la houle, il faut utiliser un appareillage complémentaire (s).

Depuis longtemps déjà, lors de la détermination des hauteurs de marées à l'aide d'appareils du type FAVE, on avait observé qu'un manomètre placé au fond de la mer restituait également les variations de pressions dues au passage de la houle.

Mais les mesures absolues effectuées avec de tels appareils ne peuvent être utilisées pour déterminer le creux de la houle, car celui-ci n'apparaît que comme un élément annexe de la mesure. Il en résulterait une précision très insuffisante. Ainsi, si l'appareil est immergé par $30 \mathrm{~m}$ de fond et si la houle a une hauteur de I mètre, la mesure de cette dernière ne représente que le trentième de la mesure absolue, en admettant que les pressions hydrostatiques dues à la houle se transmettent intégralement au fond, ce qui n'est d'ailleurs pas le cas.

D'où l'idée, exploitée simultanément par l'Amirauté anglaise et le Laboratoire de Chatou, d'employer un dispositif manométrique diftérentiel, dont une face est soumise à la pression correspondant au niveau statique moyen, et l'autre à la pression totale.

Le dispositif qui utilise un manomètre est en définitive moins coûteux que l'appareil d̀ ultra-sons, il a une plus grande sensibilité et s'accommode beaucoup mieux que celui-ci d'un récepteur autonome, mais ses indications doivent être corrigées d'un facteur tenant compte de la profondeur d'immersion et de la période de la houle. Ce facteur peut être déduit de la théorie de la houle. La première approximation de cette théorie indique que le creux en surface est égal à la variation de pression au fond multipliée por le cosinus hyperbolique de $2 \pi \mathrm{H} / i$, $H$ étant la profondeur d'immersion, et $\lambda$ la lon. gueur d'onde de la houle au point considéré.

Cette formule a été vérifiée par SEIWELL ( $\left.{ }^{0}\right)$ au moyen de mesures directes comparées avec ies mesures manométriques. II trouve qu'il faut multiplier le creux calculé par le coefficient 1,35. Ce coefficient devrait d'ailleurs être corrigé pour

(8) C'est un problème étudió actuellement au Labo. ratoire de CHATOU. Le copteur de direction sera relié, soit à la terre par le câble du manomètre (qui comportera alors 6 conducteurs): soit à un enregistreur autonome du type décrit plus hout.

(9) SEIWELL H.-R. : Investigation of underwater pressure records and simultonecus see - surfoce partems. Trons. Amer. Geophys. Union, v. 28, p. 722-724, 1947. 
tenir compte du fait que SEIWELL prend pour $\lambda$ la longueur d'onde de profondeur infinie. Je crois qu'il y aurait intérêt à recommencer ces expériences en comparant pendant une longue période les mesures faites au sondeur à ultrasons avec les relevés manométriques.

\section{DESCRIPTION DU MANOAETRE ENREGISTREUR DE PRESSIONS SOUS-MARINES TYPE CHATOU}

(Fig. 1 et 2)

La partie immergée de notre manomètre comporte un trépied sur lequel viennent s'installer !e dispositif compensateur de pression moyenne et le manomètre différentiel lui-même; le poids de l'ensemble est de l'ordre de $120 \mathrm{~kg}$. Les fils électriques sortis du manomètre aboutissent $\dot{a}$ une boite de jonction fixée sur le trépied, où ils sont raccordés aux fils du côble sous-marin qui conduit à terre.

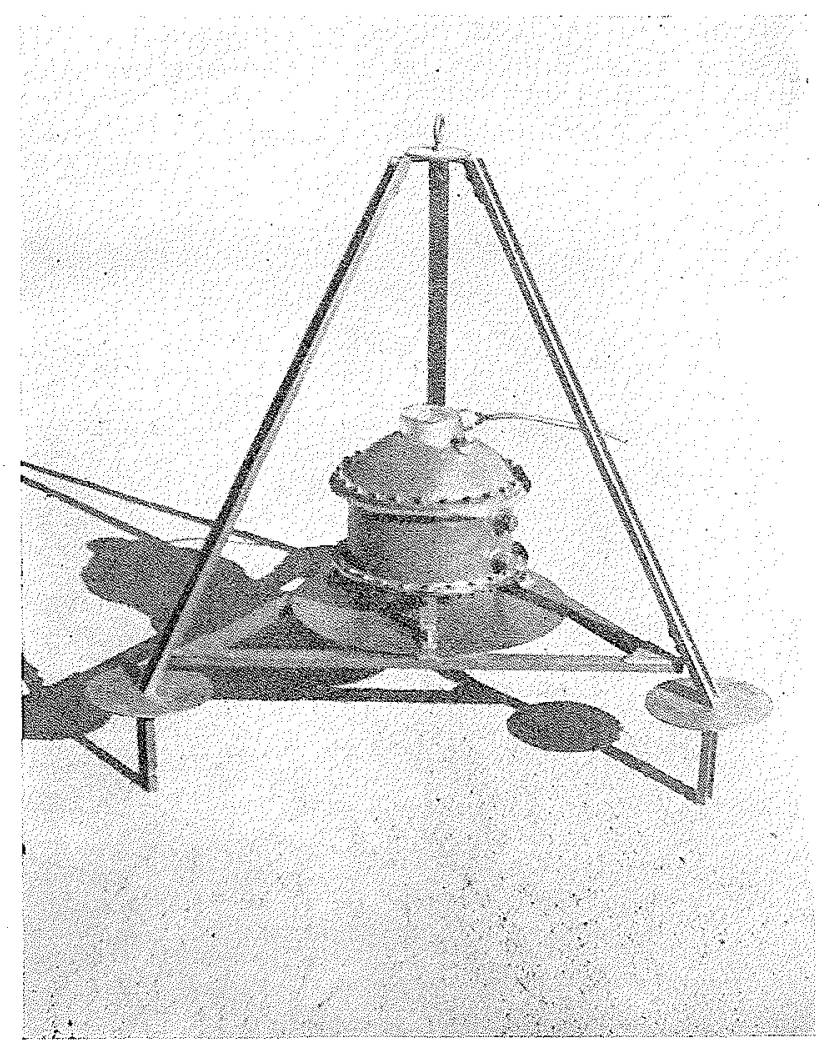

Fig. 1

\section{1. - Compensateur de pression (voir figure 2)}

Ce dispositif est destiné à transmettre la pression moyenne de la mer à une chambre d'air en liaison avec une face du manomètre diffe

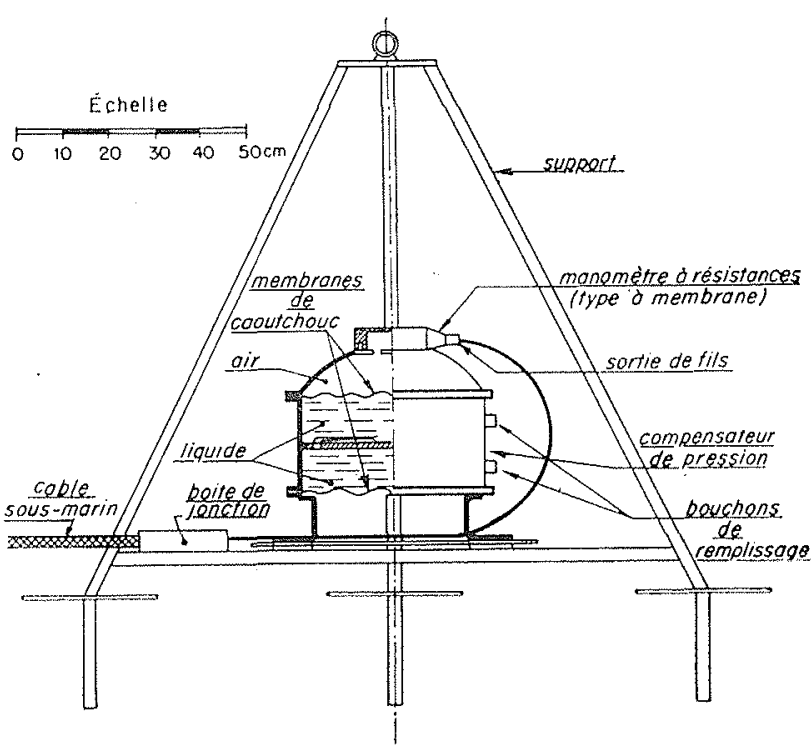

Fig. 2- SCHÉMA GÉNÉFAL

rentiel. La pression est transmise directement à travers une membrane de caoutchouc souple à un premier compartiment contenant un liquide. C.e compartiment communique, par l'intermédiaire d'un tube fin et long, avec un deuxième compartiment, lui aussi rempli de liquide, dont 1o pression est transmise, par l'intermédiaire d'une membrane de caoutchouc souple, à la chambre d'air qui lui est accolée.

Si l'on immerge l'appareil dans une mer calme, à une profondeur déterminée, lo pression du rrilieu s'établira dans la chambre d'air par l'in. termédiaire des deux compartiments remplis de liquide et du tube fin qui les fait communiquer. Les variations lentes de la pression, comme par exemple les variations dues à la marée, seront bien transmises à la chambre d'air, mais non les variations rapides qui seront absorbées par lo résistance visqueuse qu'oppose le tube fin au passage du liquide. Les dimensions que T'on donnera à ce tube dépendront de la période des variations de pression que l'on veut amortir, de la viscosité du liquide employé, et du volume et de la pression absolue de la chambre d'air.

\section{1. - Monomètre diftérentiel.}

Nous avons étudié plusieurs types de manomètres différentiels électriques destinés à mesurer la différence entre la pression totale de la mer et la pression moyenne fournie par le compensateur.

Nous nous sommes fixés en définitive sur deux types de manomètres. L'un comporte une membrane métallique taillée dans la masse d'un 
jet de bronze et séparée en secteurs par des fraisures, l'étanchéité étant assurée par une plaque de caoutchouc collée. Les déformations cie la membrane sont mesurées par des résistances extensométriques (strain-gauges) collées en des points judicieusement choisis, de façon à former un pont de Wheatstone qui est désé-

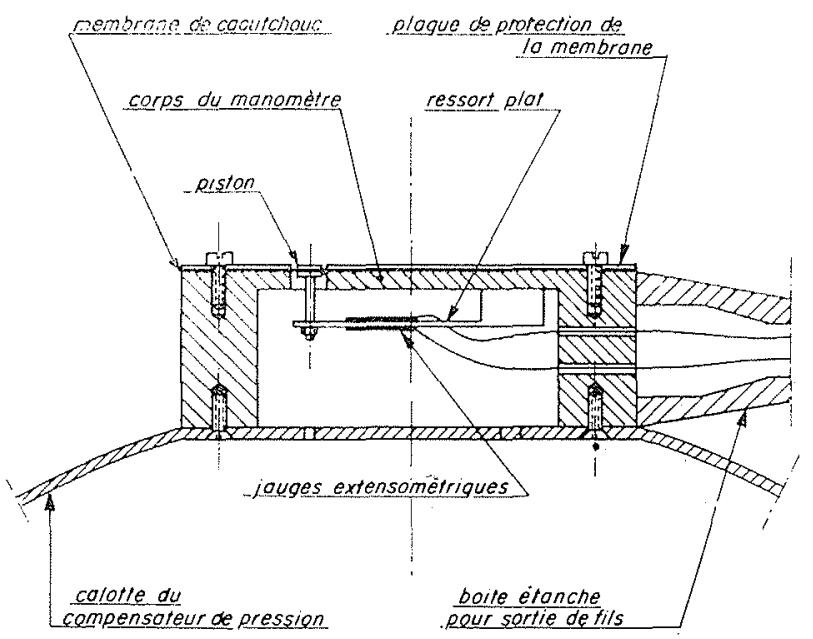

Fig. 3- MANOMETRE OIFFERENTIEL quilibré dans un sens ou dans l'autre selon le signe de la différence de pression à mesurer.

Ce type de manomètre convient mieux que le suivant aux mesures de marée. Pour ces mesures, on fera le vide à l'intérieur du manomètre de façon à éviter les erreurs dues aux variations de température et, naturellement, on i'utilisera pas de dispositif compensateur de pression. Dans l'autre type de manomètre (figure 3), la différence des pressions agit sur un piston sans frottements, l'étanchéité étant cssurée par une membrane en caoutchouc souple collée. L'effort exercé sur le piston est mesuré par un ressort plat taillé dans la masse d'une tôle épaisse de duralumin. De chaque côté du ressort sont collées deux jauges extensométriques formant un pont de Wheatstone.

L'intérêt de l'utilisation des strain-gauges est que, avec le même appareil, on peut mesurer des variations de pression d'amplitudes très diverses avec une bonne précision. En effet, si ie ressort est calculé pour permettre la mesure de houles de quatre mètres avec une déformation de $1 / 1000^{\circ}$ des fibres extrêmes, on pourra mesurer une houle de $10 \mathrm{~cm}$ avec une précision de l'ordre de $1 \%$.

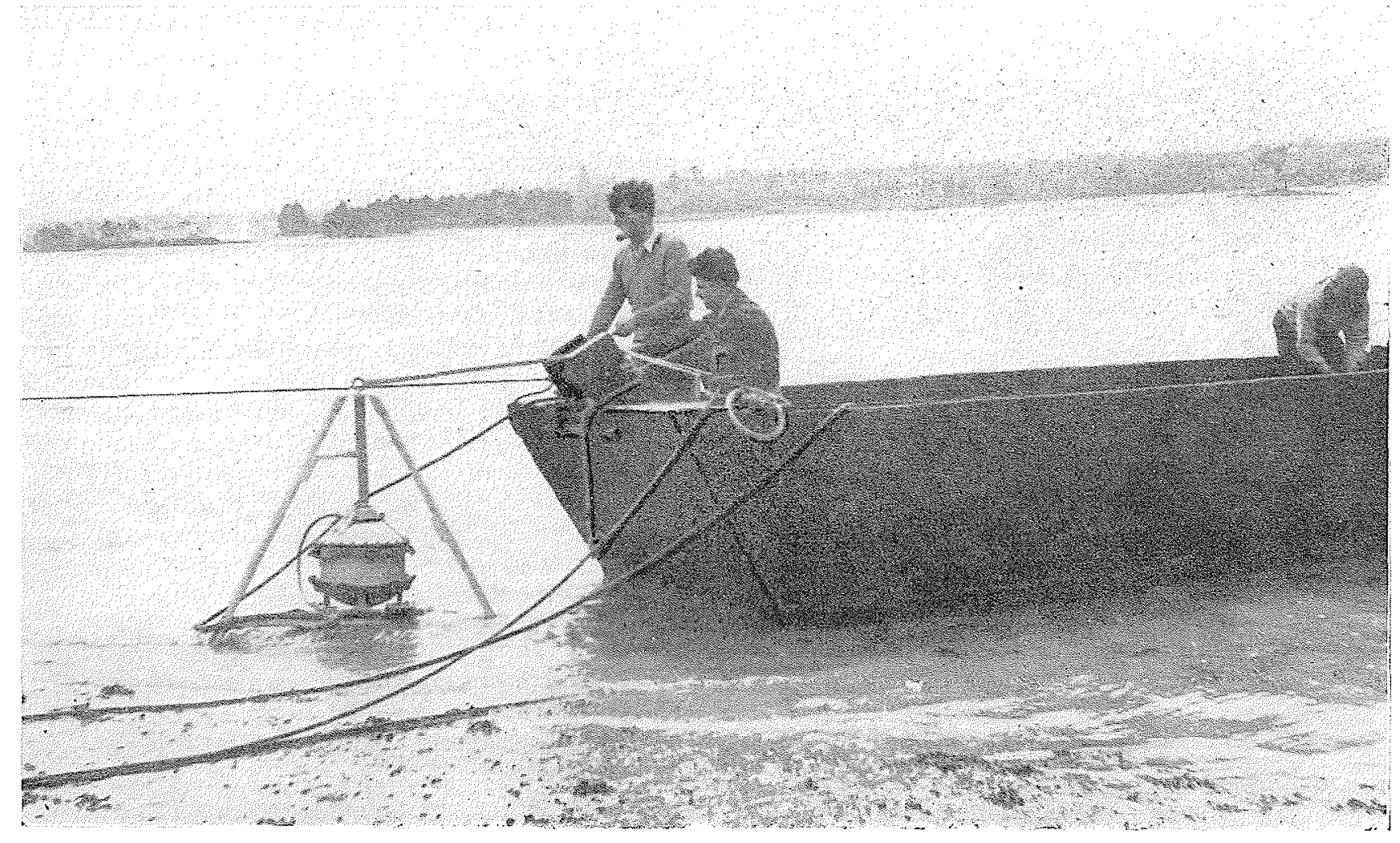

Fig. 4 


\section{1. - Dispositif récepreur ef enregistreur.}

Nous l'avons fait étudier par une maison spécialisée dans la construction d'équipements pour mesures extensométriques par "strain-gauges".

Cet équipement, qui peut être alimenté par le courant du secteur ou par un convertisseur rotatif à partir d'accumulateurs, peut entraîner directement un enregistreur graphique.

II est prévu avec une fréquence porteuse basse $(300 \mathrm{p} / \mathrm{s})$ pour permettre l'utilisation de cables longs. La tropicalisation des circuits et le maintien de la constance de la température inté rieure par un thermostat permettent de l'utili. ser dans les pays tropicaux.

Avec ce dispositif, l'erreur que l'on peut faire provient en pratique uniquement de l'appareil enregistreur. La sensibilité est de l'ordre du $1 / 1000^{\circ}$ de la différence de pression maximum que peut supporter le manomètre différentiel.

On peut utiliser soit un enregistreur graphique, soit un enregistreur photographique.

Pour l'enregistrement d'ondes longues (seiches), on peut intercaler entre deux circuits du récepteur un filtre qui dérive une grande partie des courants correspondant aux ondes plus courtes (houle).

\section{ESSAIS DE L'APPAREIL DANS L'ESTUAIRE DE LA RANCE}

Nous avons mouillé un manomètre du type à piston dans une anse de l'estuaire de la Rance pour des essais de longue durée.

La figure 4 montre le manomètre sur la plage avant son mouillage. Les $200 \mathrm{~m}$ de câble qui le relieront à la terre sont lovés dans le bac. Les cppareils récepteurs étaient dans un abri, à terre, et fonctionnaient sur accus (figure 5).

La figure 6 montre un enregistrement effectué dans ces conditions. L'échelle des temps est $1 \mathrm{~cm}$ pour 10 secondes, celle des pressions 19 divisions pour $2 \mathrm{~cm}$ d'eau de mer (sensibilité maximum). La houle enregistrée de période 10 secondes environ était pratiquement invisible à l'œil, comme on peut le voir sur la figure 7 , où l'on ne peut distinguer que les rides courtes créées par le vent.

\section{CONCLUSION}

L'idée qui nous a guidé dans le choix des principes de fonctionnement du manomètre à houle était d'obtenir un appareil tel que sa partie réceptrice soit utilisable telle quelle pour la plupart des mesures océanographiques. Je crois que les strain-gauges répondent bien à

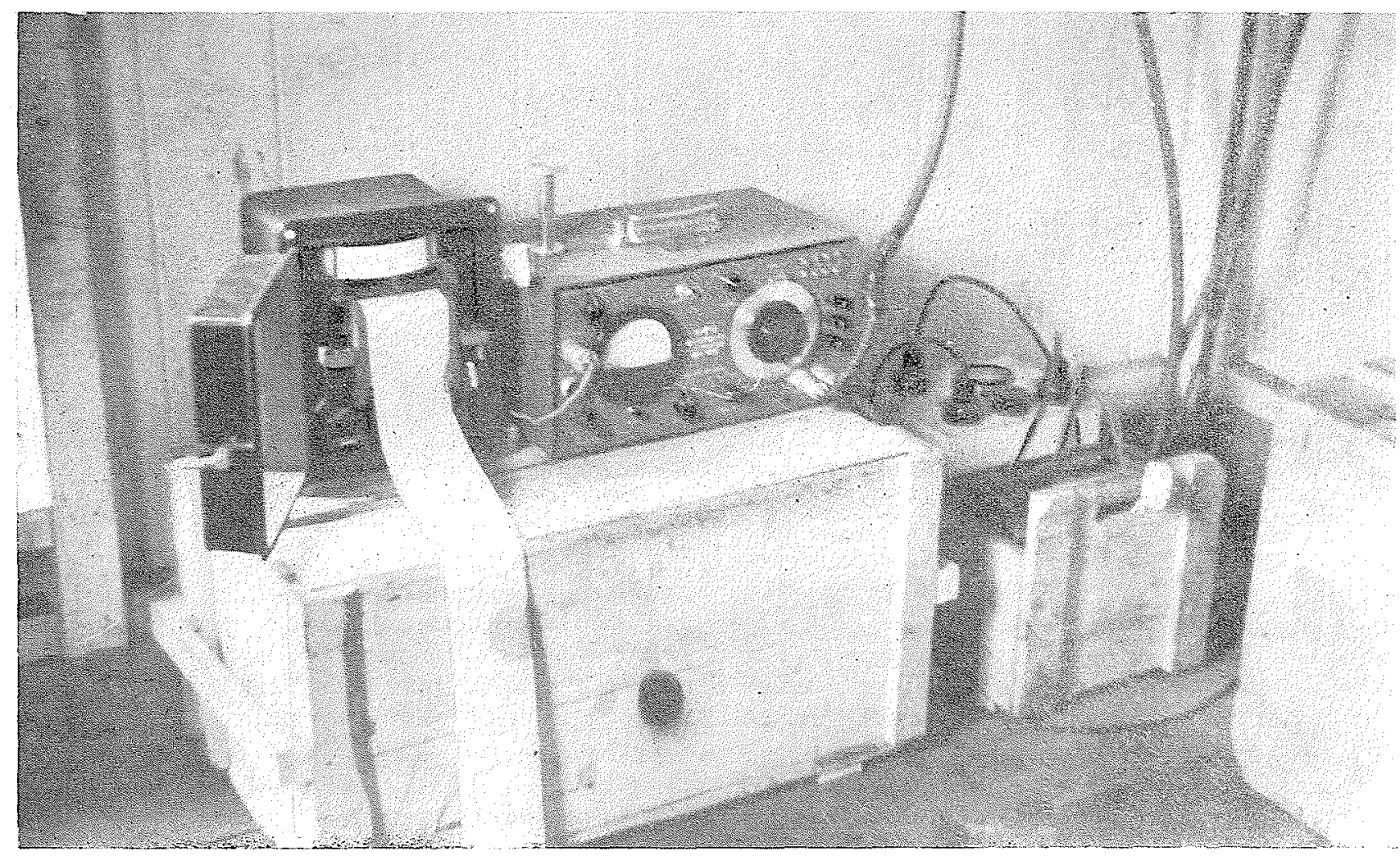

Fig. 5 


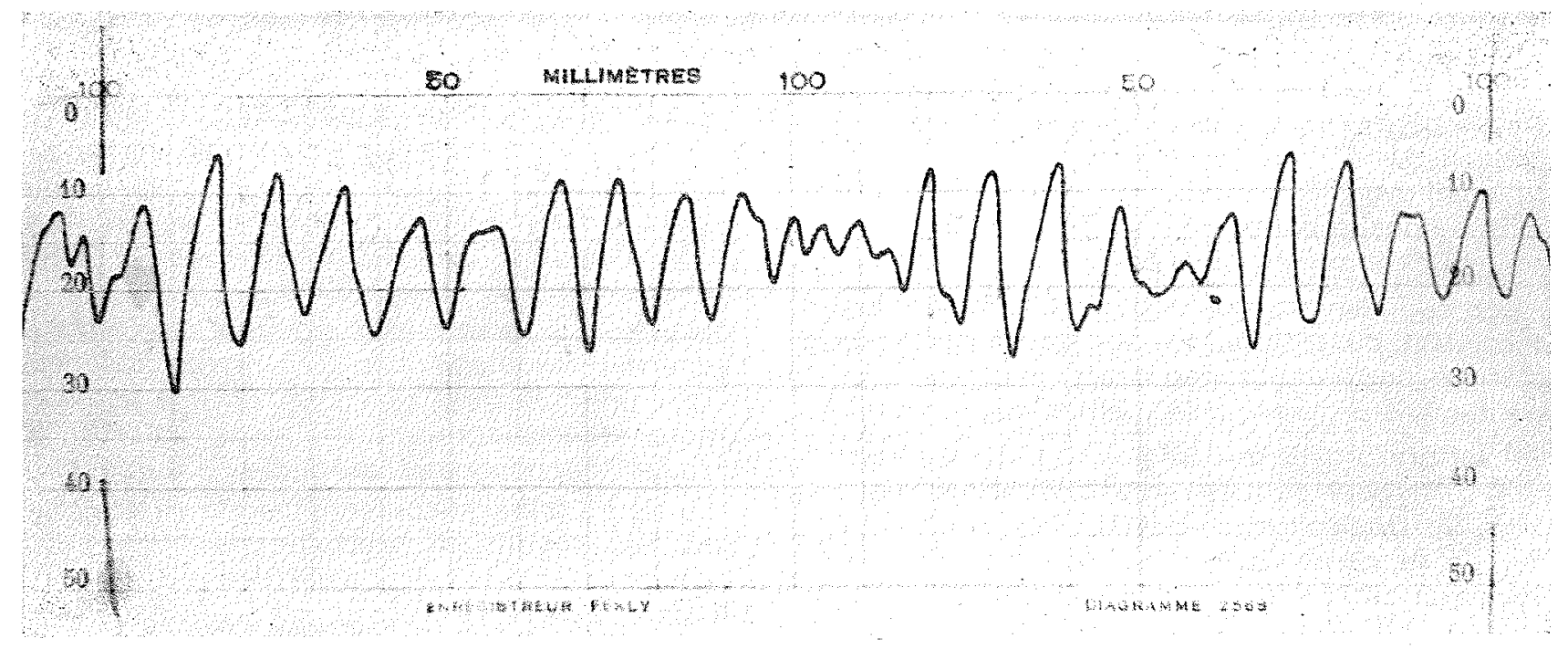

Fig. 6

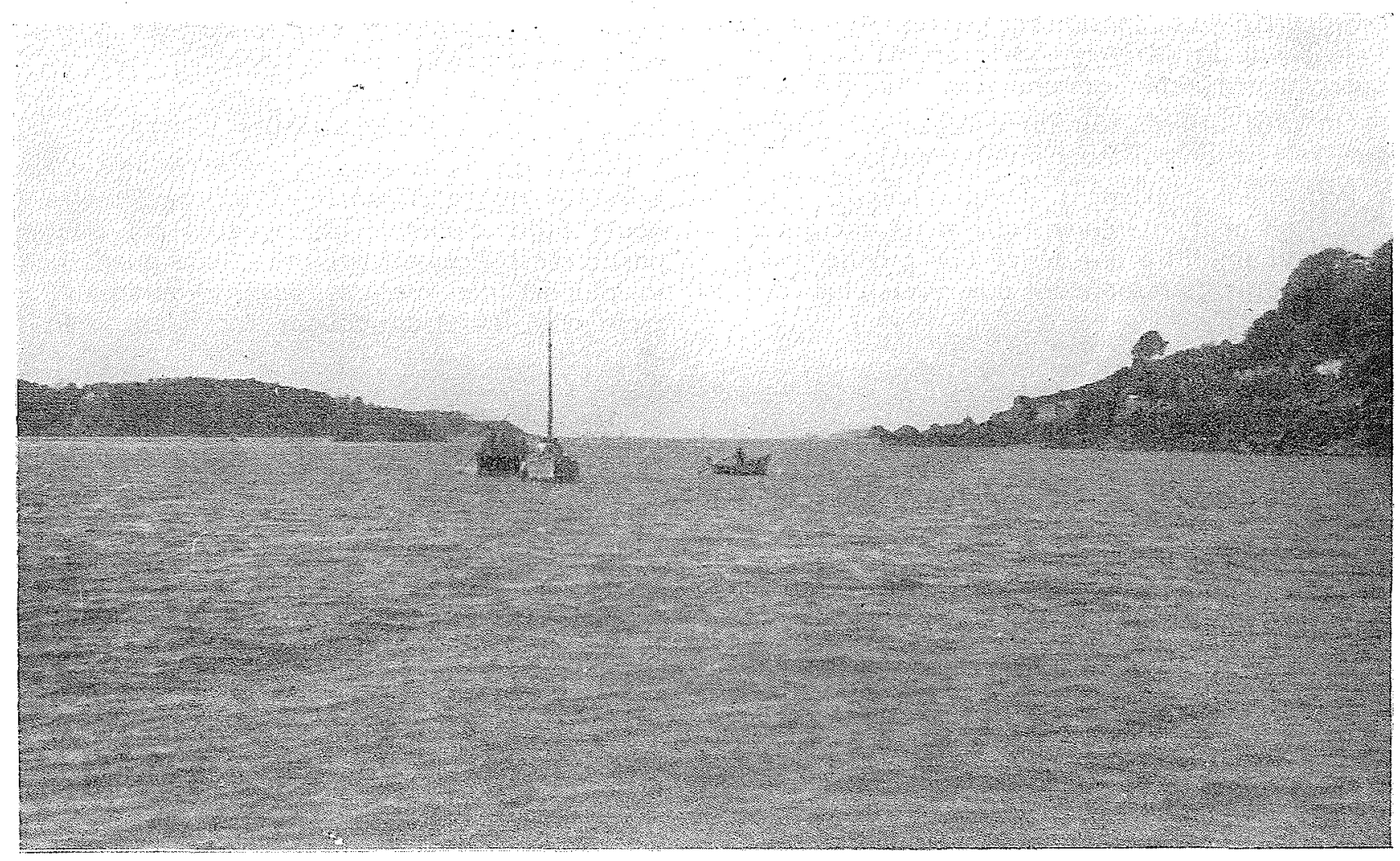

Fig. 7

cette condition. On pourra les utiliser pour réaliser des capteurs de pression (houles, seiches, marées), de courants, de température. La précision qu'elles permettent est très grande. Par exemple, avec un manomètre calibré pour enregistrer des houles maxima de $4 \mathrm{~m}$ de creux, on mesurera des houles de $10 \mathrm{~cm}$ à $1 \%$ près.

D'autre part, les deux types d'enregistreurs que nous envisageons, l'enregistreur à terre relié au capteur par un câble et l'enregistreur autonome, couvriront la plupart des besoins. Les mesures a partir de navires océanographiques pourront utiliser les mêmes capteurs et des enregistreurs de l'un ou l'autre type. Ces enregistreurs pourront d'ailleurs fonctionner moyennant de très légères modifications avec des capteurs basés sur un principe de mesure autre que les straingauges.

Nous pensons ainsi crriver à un matériel de mesures océanographiques pratiquement standard, c'est-à-dire à des appareils polyvalents relativement peu coûteux. 


\section{DISCUSSION}

$M$. le Président de ROUVILLE remercie M. VALEMBOIS, rappelle les raisons de l'interêt de l'opporeil décril et olivre la discussion en la sériant comme suit:

fo Discussion sur l'apporeil lui-même;

$2^{\circ}$ Discussion sur la formule.

Il souligne, d'autre part, que les exécutants de rravaux maritimes sont surtout intéressés par la connoissance des plus fortes houles à craindre, qu'on ne peut déceler qu'au moyen de mesures faites pendant une irès longue période.

\section{f' DISCUSSION SUR L'APPAREIL.}

M. LARRAS signale les cifficultés à vaincre pour arriver - une utilisation pratique de l'apporeil decrit, dont ie principe lui parait excellent : résistance aux éléments physiques, chimiques et biologiques du milieu où ii est placé, étanchéité, rattrapage des jeux ò distance, etc...

Sur la suggestion de $M$. le Président, M. VALEMBOIS indique d'abord la profondeur optimum a laquelle doit être placé l'appareil : dans les grandes profondeurs, les différences de pression enregistiées seraient très faibles et on pourrait en déduire des creux erronés; dons les fonds peu profonds, au voisinage des côtes, lo houle est déformée et les courants agissent sur l'appareil. Une bonne profondeur d'immersion serait comprise entre 20 ef $30 \mathrm{~m}$.

Puis $M$. VALEMBOIS répond à $M$. LARRAS que l'apparell immergé pendant un mois dans l'estuaire de la Rance ría causé aucun des ennuis que celui-ci redoute: I'iso!ement électrique, notamment, $n$ 'a pas eu de déficience et il n'y a pas de jeu à l'intérieur de l'appareil, parce qu'i! r'y a pas d'articulation.

M. FOCH signale l'utilisation par les Allemands pendant la dernière guerre de stations météorologiques outomatiques immergées à certaines heures et émergeant à d'autres pour relever et transmettre des renseignemenis, et susceptibles de fonctionner pendant trois semaines sans qu'on les relevât. Une adaptation de l'appareil à houle dans cette voie lui semble donc à première vue possible.

M. DANEL signale que les Anglo-Américoins ont étudié et mis au point la transmission à distance, sans câbles et sans bouées, d'appareils immergés, mais n'ont rien voulu publier jusqu'à présent ò ce suje:

$M$. BESSON suggère d'associer d̀ l'appareil du fond une bouée qui lui serait reliée par câble, renfermerait un petit émetteur radioélectrique et transmettrait les indicarions du manomètre.

M. NIZERY craint que les bouées ne soient détruites ou ciéréglées pai les tempêtes, et tient pour essentiel d'ovoir un appareil constamment et entièrement immergé

M. VALEMBOIS rappelle que le Laboratoire National d'Hydraulique met au point un enregistreur autonome destirié à être mouillé auprès du manomètre. Cet opparei! serait prévu pour fonctionner pendant plusieurs mois, en enregistrant photographiquement de temps en temps loar exemple 20 minutes toutes les 6 heuresl, les indications du manomètre. Les enregistrements pourront ciussi être déclenchés lors d'une tempête.

M. BIESEL signale l'étude par les Anglais d'un appareil ì quartz entièrement fermé, cylindrique, qui prêterait moins le flanc aux critiques de M. LARRAS. Cependant il ne sait pas si les résuliats obtenus avec cet appareil ont été satisfaisants.

Il souligne également que ce type d'appareii de mesure de la houle (les manomètres immergés) présente un intérêt considéroble, car il permet cie faire des mesures et d'établir des statistiques d'une haute valeur pratique et scientifique.
Ces statistiques sont d peu près inexistantes actuellement en France, alors que les Anglais et les Américains en ont etabli depuis plusieurs anrees, ce qui a permis par exemple a SEIWELL de rouver le coefficient empirique de lo formule indiquée por $M$. VALEMBOIS.

$M$. BIESEL pense donc qu'it est très intéressant que le Laboratoire de Chatou ait mis ou point un apparei! francais, d'autant plus que cet appareil est original en ce quil présente une fuite permanente en milieu liquide, alors que les appareils américains e: anglais presentent soit une fuite intermittente, soit uns fuite permanente.

M. LARRAS donne des précisions sur le nombre d'appa. reils construits et vendus por les Anglais.

M. GARIEL soulione le coût du câble, trés télevé vis-ó-vis de celui de l'oppareil et, de ce fait, opprouve l'idée suivie par Chatou d'enregistrer sur place par photographie.

M. VALEMBOIS parle de l'appareil anglais mouil!é dans 'estuaire de la Rance en même temps qu: celui de Chatcu, et de ses inconvénients par rapport a ce dernier: il n'a qu'une sensibilité, nécessite plus de fils, et son enregistrement se fait uniquement par photographie. Par contre, il use moins de courant que l'oppareil L.N.H. actuel.

M. LARRAS pense que les oppareils immergés doivent ratiquement être prévus pour une autonomie de fonctionnement supérieure à un mois, car ils sont souvent inaccessibles plus longtemps, rotamment en hiver (quatre mois) : ils doivent aussi présenter pendant ces immersions prolongées une robustesse et une sécurité qui entroinent oux prix très elevés mentionnés tout à l'heure: noromment le plus grand nombre de fils des appareils anglais, au demeurant conçus suivant la même schéma, est dû au souci de ménager un fil de rechunge et un fil de commutation de cer:ains organes doubles.

M. VALEMBOIS, qui a utilisé l'appareil angla:s, maintient que les six fils sont nacessaires à son fonctionnement.

$M$. Ie Président conclut que, de toute façon, un ou deux fils de plus ne grèvent pas énormément le prix du cáble.

M. GOUGENHEIM signale que dans l'étudic de la houle, on doit envisager aussi la ciéviation de propagation et la ciécomposition du phénomène dans le cas des houles stiperposées.

M. le Président répond à $M$. GOUGENHEIM que cela fait songer à son apoareil pour lo production des maréas. grâce auquel on décomposcit les ondulations enregistrées, $e_{i}$ souhaite qu'on arrive à un oppareil semblable pour la décomposition de la houle an ondes élémentaires.

M. VALEMBOIS répond que ces questions vont maintenant être étudiées par le Laboratoire National d'Hydrau. lique.

M. BIESEL indique le dispositif itudis far la Marine Anglaise pour repérer la airecion de la houle: bobine magnétique plate mise horizantalement dans l'eau dans une lentille profilée qui porte trois électrodes a sa périphérie : ¿es mesures aifférentielles des différences de potentiel induites entre ces trois points dans l'eau en mouvement permettent de déterminer ia direcion de la vitesse de l'eau. L'appareil utilise une méthode analogue à celle exposée par $M$. REMENIERAS dans sa communication à la réunion du 24 novembre 1947 «SUR LA POSSIBILITE DE TRANSFORMER DIRECIEMENT EN ENERGIE ELEC. TRIQUE UNE PARTIE DE L'ENERGIE D'UNE VEINE FLUIDE $\gg$ (")

(1) Voir "Mémoires et Travaux *, année 1948, no 1 (page 74) et. no 11 (page 115 ). 
M. VANTROYS tire les conclusions suivantes:

I: L'enregistreur doit être, autont que possible, eicigne de la zone la plus agitée, c'est-à-dire de la surface. La houle s'amortisjant exponentiellement avec la profondeu:, it y a intérêt j plocer cet enregistreur sur le fond, et plus les fonds sont grands, mievix cela vaut (pour la protection de l'enregistreur\}. Ceci est un inconvénient pour un appareil du type "monométrique ", tel que celui décrit par M. VALEMBOIS, car i! y perd à la fois sa sensibilité et sa fidélité len effet, les oscillations de pression s'amortissent avec la profondeur et l'incertilude croit sur la valeur de ia formule transformant oscillations de pression sur le fond er. dénivellations sur la surfacel. Par contre, un apparei! du type "sondeur inversé ", peut fonctionner très normalement ò une grande protondeur, sa sensibilité restant indépendante de la profondeur.

$2^{\prime}$ Les indications de l'appareil enregistreur doivent être transmises en évitant la zone agitée (superticieile), ce qui condamne, ou tout au moins rend difficile à mettre au point d'une façon sûre la retransmissicn par poste radio situé dons une bouée. Une bouée trop légère ou trop légèrement amarrée serait enlevée par la tempête. Par contre une grande bouée du type "Phares et Balises », et l'im. posant mouillage (chaine et corps mort) qui lui est associé, risqueraient de réagir dangereusement sur l'enregistreur luimême; personnellement, $M$. VANTROYS réalise mal comment pourraient être protégés contre une usure rapide les câbles reliant l'enregistreur fixe à la bouée (mobile). C'est pourquoi, malgré son prix élevé, il ne voit pas actuellement d'autre solution vraiment pratique que celle de relier l'enregistreur à la terre par un câble armé atterrissont dans une crique abritée. C'est la solution adoptée dans une station d'enregistrement de la houle que la SOCIETE D'ETUDES POUR L'UTILISATION DES MAREES, vient de mettre en place ò Chousey.

\section{$2^{\circ}$ DISCUSSION SUR LA FORMULE}

M. LARRAS dit que l'oppareil tel qu'il a été décrit, devrait être complété par un analyseur qui permette de connaître isolément, par l'analyse des périodes des houles élémentaires, le $\cosh \frac{2 \pi \mathrm{H}}{i}$ correspondant à chaque $\delta$ he mesuré par le manomètre; cet appareil devrait de plus pouvour être déclenché à volonté et à distance, de manière à n'enregistier que des houles ayant des composantes porticulièrement intéressantes et à ne pas s'embarrasser d'un enregistrement continu qui serait trop long à dépouiller et dont la plus grande partie ne présenterait pas d'iniérêt. L'appareil ainsi complété permettrait de fixer, pour chaque endroit de mesure, un coefficient moyen permettant de remplacer le signe $i$, coefficient que les Américains ont pris globalement égal à 1,35.

D'autre part, selon $M$. LARRAS, les composantes évanescentes des mesures faites en profondeur forment une somme qui n'est pas négligeable bien que difficile à esti. mer, et qui peut entrainer une erreur de la moitié ou du quart dans la détermination de to houle ainsi que l'ont prouvé des mesures faites parallèlement en surfoce.

L'appareillage complémentaire nécessité par l'analyseur, les protections de l'apporeil lui-même et dés cábles à la sortie de mer, le raccordement avec le poste d'observaion situé à terre dons un bâtiment sont autant de frais d'installation qui rendent très coûteux le disposifif complet, malgré le prix relativement modique de l'appareil.

M. le Président pense quo la prise en considération d'une ou deux des houles composantes donnerait une bonne approximation en même iemps qu'une simplification des calculs, les autres houles pouvant être traitées par un coefficient à déterminer.

M. BIESEL s'assccie aux remarques que $M$. LARRAS vient de formuler et il ajoute que, non seulement l'ampliiude mesuree subit des modifications dont la formule classique ne rend pos comple, mas qu'il en est de même pour la période, ce qui est beaucoup plus urprenan:

1] semblerait en effet ulue l'apporeil immerge devrait recueillir les tluctuations de pression correspendant au passage des crêtes en même temps que l'on voit celles-ci Eovancer en surface, c'est-a-dire avec la même frécuence. Or ce n'est pos tout dे fait vroi, parce que la houle rédlo n'a pas une fréquence pure, mais résulte en quelque sorte d'une composition de houles et a donc un ceriain spectre de fréquence. Ce spec're a " grosso modo " l'allure d'une courbe en cloche et la période observèe pratiquement en surface est une période moyenne qui se situe ò peu près vers le sommet de cette courbe.

Or si on se place à une certaine profondeur on enregistre une perturbation qui correspond du spectre décalé vers les basses fréquences, car cette courbe est très réduite du côté des fréquences élevées et au contraire mieux conservée du cốé des fréquences bassej. Ainsi la fréquence opporento de l'enregistrement est queiquefois notablement docissé.

M. VALEMBOIS reconnait l'intérêt de l'analyse des fréquences qu'il a étudiée par ailleurs mais ne croil pas quo l'analyse complète soit. toujours absclument nécessaire. D'autre part, il pense que le spectre de fiéquences est composé de plusieurs bandes distinctes, et que seule la période moyenne de chaque bande es: un peu déplacée vers les longues périodes, l'écart considérable observé par SEIWELL et signalé par M. BIESEL sur la valeur de la période moyenne provenart p'utôt de l'extinction complète de bandes à courte période.

M. MICHE cbserve que la formule indiquée est celle de première approximation et que les approximations d'crire supérieur ne sont plus négligeables pour les houles assez cambrées comme les houles de tempête. La valeur du coefficient proportionnel est augmentée de ce fait au delò de l'unité même pour une houle (ou un clapotis). théoriquement purs; en outre, elle n'est plus constante mais dépend de la cambrure et de la longueur d'onde rapportée à la profondeur. Notomment, pour les ondulations de forme limite sur le point de déferler, le coefficient dépasse 1,14 et peut atteindre, dans certains cas, pour des houles courtes, jusqu'à 1,50 environ.

M. LARRAS attire l'attention sur les changements de fréquence en passant sur tos houls-fonds, signalés par $M$. le Président BARRILLON et qui sont loin d'être négiigzables, et maintient son point de vue sur iu nécessité de faire toujours l'analyse de la houle, afin d'éviter des éva. luations trop éloignées de la réalité, étant bien entendu d'ailleurs que l'évoluation, même faite avec tout le soin voulu, sera toujours très grossière, par suite, noiamment, do la différence entre ce qu'on appelle « la creux en surface » et la hauteur réelle d'après laquelle les ouvrages devraient être calculés.

$M$. le Président croit quion se trompe moins pour l'svatuation des très grandes naules gue l'on observe en mer, parce que l'on monte dans le mot pour afflecirer l'horizon que pour les houles moyennes bosées sur une distance verticale entre creux ot crèes.

M. PETRY décrit comme suit un autre dispositif de mesure de la houle:

"Si l'on désire la courbe représentant en un poin lo haureur d'eau au-dessus du fond de lo mer, en fonction du temps, on peut obtenis directement cetle courbe par divers dispositifs:

L'un d'eux consiste ò compter, visuellement ou au moyen d'un kymatogrophe (2), le nombre de décimètres alternotivement émergeont ou immergés le long d'une

2) Voir compte rendu des "Annales des Ponte of Choussées" 1938, VII, pages 66 aे 72 
balise constituée d'un tube métallique vertical scellé dons une embase posée sur le fond et ensuite chargée de viellas chaines.

Deux balises de ce genre, utilisees devant Dicpoe de 1932 à 1939, ont résisté aux tempêtes, bien que les lames déferlent souvent autour d'elles. Un kymarogrophe, instalk sur la parci de la jetée Ouest, a lonctionné des années durant sans erreur et sans incidant.

Un autre moyen est d'immerger au fond de la mer dans une boite étanche, un sondeur à ultra-sons installé sens dessus dessous, pour mesurer et enregistrer les variations de la distance qui le sépare de la surface de l'eau.

11 serat bon de metre en cuvre, en une staion d'assais l'in ou l'autre de ces dispositifs, conjointement avec les divers appareils enregistrant les variations de la pression au fond afin de contrôler à la fois le bon fonctionnement de ces divers appareils et l'exactitude des calculs qui donnent la variation de la hauteur d'eau à partir de la variation de la pression au fond. "

Répondant j̀ une question de $M$. le président Barrilion, $M$. le président de Rouville indique que le coút de l'olivroge décrit par $M$. PETRY est diun ordre de grandeur très inférieur à celui des appareil's ang'o-américains déjà mentionnés.
M. BIESEL signa'e à propos des mesures directes de to houle, que cellesmci ont presque tcujours été faites à titre de vérificolion par les premers utilısateurs des manomètres immerges. Cetie mesure etait faite soit à l'aide d'un sondeur ultra-sonore inversé soit par stéréophotogramméirie. Cela a eté fait en particulier par SEIWELL qui filmait une boué et relevait les iridications du manomètre simultanément; la synchronisation des deux enregistrements était ob:enue par l'explosion d'une charge de dynamite qui donnait une gerbe visible sur le film el un top enregistro par le manomètre.

M. NIZERY répond aux deux principales critiques faites à l'appareil presenté par $M$. VALEMBOIS par les deux mises au point suivantes:

$1^{\circ}$ Coùt: 2 milions envircn pour une installation placéc à 2.000 mètres de la côte (bien inférieur a celui qui a été avancé pour un appara!l étranger).

$2^{\circ}$ Sûreté de la réponse: Comme les appareils de mesure en général, celui-ci doit ėtre étalonné par comparaison avec les résuliats observés parallèlement au moyen d'un qutre procédé : ultra-sons de préférence. Une fois taré, l'appareil peut donner des résultats sur lesquels on peuí se fonder.

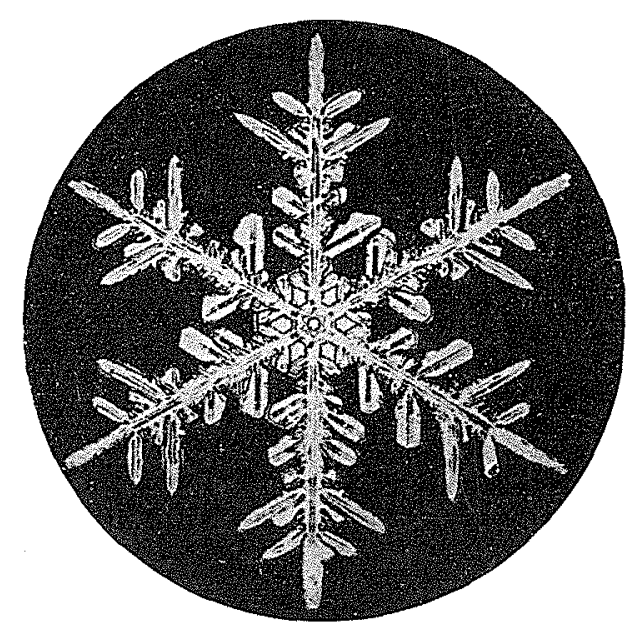

